\title{
On Routing with Guaranteed Delivery in Three-Dimensional Ad Hoc Wireless Networks*
}

\author{
Stephane Durocher ${ }^{\dagger}$
}

\author{
David Kirkpatrick ${ }^{\ddagger}$
}

Lata Narayanan ${ }^{\S}$

June 3, 2008

\begin{abstract}
We study the problem of routing in three-dimensional ad hoc networks. We are interested in routing algorithms that guarantee delivery and are $k$-local, i.e., each intermediate node $v$ 's routing decision only depends on knowledge of the labels of the source and destination nodes, of the subgraph induced by nodes within distance $k$ of $v$, and of the neighbour of $v$ from which the message was received. We model a threedimensional ad hoc network by a unit ball graph, where nodes are points in three-dimensional space, and for each node $v$, there is an edge between $v$ and every node $u$ contained in the unit-radius ball centred at $v$.
\end{abstract}

The question of whether there is a simple local routing algorithm that guarantees delivery in unit ball graphs has been open for some time. In this paper, we answer this question in the negative: we show that for any fixed $k$, there can be no $k$-local routing algorithm that guarantees delivery on all unit ball graphs. This result is in contrast with the twodimensional case, where 1-local routing algorithms that guarantee delivery are known. Specifically, we show that guaranteed delivery is possible if the nodes of the unit ball graph are contained in a slab of thickness $1 / \sqrt{2}$. However, there is no $k$-local routing algorithm that guarantees delivery for the class of unit ball graphs contained in thicker slabs, i.e., slabs of thickness $1 / \sqrt{2}+\epsilon$ for some $\epsilon>0$. The algorithm for routing in thin slabs derives from a transformation of unit ball graphs contained in thin slabs into quasi unit

${ }^{*}$ Research for this work was supported by NSERC. Some of these results appeared in preliminary form at the International Conference on Distributed Computing and Networking [14].

${ }^{\dagger}$ Cheriton School of Computer Science, University of Waterloo, Waterloo, Canada, sdurocher@cs.uwaterloo.ca

$\ddagger$ Department of Computer Science, University of British Columbia, Vancouver, Canada, kirk@cs.ubc.ca

$\S$ Department of Computer Science, Concordia University, Montréal, Canada, lata@cse.concordia.ca disc graphs, which yields a 2-local routing algorithm. We also show several results that further elaborate on the relationship between these two classes of graphs. keywords: ad hoc networks, unit ball graph, routing, distributed algorithms, quasi unit disc graph

\section{Introduction}

Mobile ad hoc networks (MANETs) have been the subject of intensive study over the last decade. Communication between different nodes in a MANET is achieved by means of a multi-hop routing protocol, which dictates how a packet from a source node should be forwarded along the edges of the network to a given destination node. Although a straightforward flooding algorithm is possible, such a strategy has obvious drawbacks, including high traffic loads [30], repetitive behaviour (if networks nodes are memoryless), and requiring knowledge of a lower bound on the diameter of the network to ensure both termination and delivery. In this paper we consider singlepath routing algorithms.

Many routing algorithms for MANETs model the network as a two-dimensional geometric graph [17, $24,25,30]$. This does indeed capture a large number of possible application scenarios for ad hoc networks, where nodes might be vehicles moving through city streets or some other terrain. However, there is increasing interest in applications where ad hoc and sensor networks may be deployed in threedimensional space, such as in an ocean, the atmosphere, or in a building $[5,18]$. For example, underwater ad hoc and sensor networks that perform ocean column monitoring would require nodes to be placed at different depths in the water, creating a threedimensional network [4]. In this paper, we study the problem of routing in three-dimensional ad hoc networks, and the extent to which they differ from twodimensional ad hoc networks from the perspective of 
routing protocols. In brief, our results show that the two settings are indeed quite different.

Two-dimensional ad hoc networks are usually modelled as unit disc graphs (UDG). Every node in a UDG can be mapped to a point on the plane, in such a way that any two nodes at distance at most one are connected by an edge. In other words, a node $v$ is connected to every node $u$ occurring within the disc of radius one centred at $v$. The unit disc centred at a point represents the transmission range of the corresponding host. In reality, however, the transmission range of a wireless node is affected by many unpredictable factors, and is unlikely to be a perfect disc. In [6], the notion of a quasi unit disc graph (QUDG) was introduced to address the issue of unstable transmission ranges. Roughly, a $d$-QUDG is a geometric graph in which any two nodes at distance at most $d$ are always connected, nodes at distance greater than one cannot be connected, and nodes at distance between $d$ and one may or may not be connected.

The ad hoc nature of the networks under consideration, and the mobility of the nodes implies that the topology of the network is arbitrary, and moreover, it changes over time. In the absence of any information about the location of nodes, routing protocols are obliged to flood control packets through the network in order to obtain information about the topology of the network [29]. However, in many cases, it is reasonable to assume that nodes do have access to information about not only their own locations, but also the location of their immediate neighbours, and correspondent nodes, via GPS and location servers. There is a large body of work on routing protocols that utilize position information in order to achieve efficiency in routing (see the surveys [17, 30]). Most of these are heuristics, and there may be graph instances on which the routing algorithm fails to deliver the packet. In greedy routing, for example, a node transmits the packet to its neighbour that minimizes the Euclidean distance to the destination [26]. In compass routing, the next node is chosen to the neighbour that minimizes the angle between itself, the current node, and the destination node [21]. In both these algorithms, the packet can get stuck in a loop, resulting in a routing failure. The only known algorithms that are guaranteed to deliver the packet are based on face routing, in which a planar subgraph of the unit disc graph is extracted locally, and then routing proceeds by traversing the faces of this planar graph that intersect the line segment between the source and destination [21]. Face routing can be com- bined with greedy routing $[9,20]$, and can be limited in space $[22]$ to achieve faster delivery times. Face routing can also be simulated on $d$-QUDGs where $d \geq 1 / \sqrt{2}$, as shown in $[6,23]$.

The natural generalization of a UDG to three dimensions is the unit ball graph (UBG), which differs from a UDG only in the fact that nodes correspond to points in three-dimensional space. Accordingly, a node $v$ is adjacent to every node within the unitradius ball centred at $v$. Similarly, the quasi unit disc graph model can be extended to a quasi unit ball graph model. All algorithms that have been proposed so far for routing in UBGs are based on heuristics $[1,2,3,15,19]$. As yet, there is no known algorithm for routing that guarantees delivery in such networks. In this paper, we address the question of what kinds of UBGs admit a routing algorithm that guarantees delivery.

The answer to this question depends on the information that is available to a routing algorithm in deciding where next to forward a packet. At one end of the spectrum are algorithms that have complete information about the entire graph, and that can store routing tables that contain next-hop information along shortest paths for every possible destination. At the other end are the so-called online and memoryless algorithms [8], where a node makes its forwarding decision based only on the labels ${ }^{1}$ of itself, the destination node, and its neighbours. Bose et al. show that there is no deterministic memoryless algorithm that is guaranteed to succeed even if the graphs are limited to convex subdivisions [7].

Routing algorithms with complete information are entirely unsuitable for the application domain of mobile ad hoc networks, with their changing topologies, autonomous nodes, and low-bandwidth wireless links. On the other hand, memoryless algorithms are far too restrictive. For example, in practice, when a node receives a message, it knows which of its neighbours sent it. Yet, it is precisely the lack of this information that makes it impossible for a memoryless algorithm to route on all convex subdivisions; the only information outside the memoryless model available to face routing, which does succeed on all convex subdivisions, is knowledge of the previous node. Similarly, it would be reasonable to allow a node knowledge of the topology of its $k$-hop neighbourhood for small and fixed values of $k$. We say an algorithm is $k$-local if a node has access to the topology of its $k$-hop neighbourhood, as well as the previous node on the path,

\footnotetext{
${ }^{1}$ In a geometric graph, a node is labelled by its coordinates.
} 
in making its forwarding decision. There has been increased recent interest in distributed algorithms that are sensitive to locality; see for example the book by Peleg [28]. Routing algorithms with information about $O(1)$ other nodes in the graph are related to $k$ local algorithms and have been studied in [21, 22]. In this paper, we restrict ourselves to routing algorithms that are $k$-local. While our algorithm for a restricted class of unit ball graphs is 2-local, the impossibility results apply to $k$-local algorithms for any fixed $k$.

\section{Our results}

In essence, we show that routing in three-dimensions is harder than routing in two dimensions. As far as routing is concerned, it is possible to "lift off" the plane to a certain extent, but not beyond. We consider unit ball graphs where nodes are contained in a slab of fixed thickness. We show that if the thickness of a slab is less than $1 / \sqrt{2}$ times the transmission radius of nodes, then there is a 2-local algorithm that guarantees delivery in the graph. Conversely, for unit ball graphs in thicker slabs, we show that if a $k$-local routing algorithm were to exist, then a $k$-local algorithm for routing would also exist for an arbitrary graph, which we show is impossible.

The algorithm for UBGs contained in thin slabs derives from the fact that such a UBG can be transformed via projection into a $d$-QUDG with $d \geq 1 / \sqrt{2}$, for which a 2-local algorithm with guaranteed delivery was outlined in [23]. We explore the relationship between UBGs and QUDGs further in Section 4. We show that neither the class of all UBGs nor the class of $d$-QUDGs is contained in the other, for fixed values of $d$. In particular, for every $d<\sqrt{3} / 2$, we exhibit a $d$-QUDG that cannot be embedded as a UBG. While it is straightforward to see that any graph can be embedded as a $d$-QUDG for small enough $d$, we show that for any fixed $d$, there are UBGs that cannot be embedded as a $d$-QUDG. Finally, our negative results on routing in UBGs contained in slabs of large enough thickness imply the non-existence of a $k$-local algorithm for $d$-QUDGs with $d<1 / \sqrt{2}$. This shows that the results of Barrière et al. [6] and Kuhn et al. [23] on routing in QUDGs are tight.

\section{Definitions}

Given a labelled, connected, undirected graph, $G=$ $(V, E)$, and two vertices, $s$ and $t$ in $V$, the problem of routing is to send a packet from $s$ to $t$ via a sequence of adjacent vertices in $G$. To this end, an algorithm for routing is implemented in a distributed manner at every node in the graph, in such a way that when the packet arrives at a particular node $u$, the routing algorithm implemented at $u$ must deterministically choose a unique neighbour of $u$ to which the packet should be forwarded. An algorithm halts once the message is forwarded to the destination vertex $t$. In this case, we say the algorithm delivers the message. We say routing algorithm $\mathcal{A}$ succeeds for a class of graphs $\mathcal{G}$ if, for all $G \in \mathcal{G}, \mathcal{A}$ delivers a message from any origin $s$ to any destination $t$ in $G$. Otherwise, we say $\mathcal{A}$ is defeated by some $G \in \mathcal{G}$.

Let the $k$-neighbourhood of a vertex $v$, denoted $G_{k}(v)$, be the subgraph of $G$ induced by vertices within graph distance $k$ from $v$ (including the corresponding vertex labels). The vertex labelling scheme should be independent of the graph; in particular, the labelling should not encode additional information about the topology of the graph or the neighbourhood of a vertex. For example, in a geometric graph, each vertex is labelled by its coordinates.

Let $\Sigma$ denote the set of possible vertex labels for a given class of graphs and let $\mathscr{P}(A)$ denote the power set of set $A$. Given a fixed $k$, we say a routing algorithm is $k$-local if it can be defined by a routing function $f: \Sigma^{4} \times \mathscr{P}\left(\Sigma^{2}\right) \rightarrow \Sigma$ with the following interpretation: $f\left(s, t, v, u, G_{k}(u)\right)$ specifies the neighbour to which node $u$ should forward the packet, provided (a) the packet was received from its neighbour $v$, (b) the source and destination of the packet are $s$ and $t$ respectively, and (c) $G_{k}(u)$ is the $k$-neighbourhood of $u$.

A $k$-local algorithm must therefore make the forwarding decision at a node $u$ based only on the source and destination nodes, its $k$-neighbourhood, and the previous node on the path. It has no additional information about the route. In particular, no memory or state information may be stored in the message other than $s, t$, and $v$, nor may the state of a vertex be modified after forwarding a message.

Given a set of points $P$ in $\mathbb{R}^{2}$, the unit disc graph induced by $P$, denoted $U D G(P)$, is an embedded graph whose vertices correspond to $P$ and for which edge $(u, v)$ exists if and only if $\|u-v\| \leq 1$. Given a set of points $P$ in $\mathbb{R}^{3}$, the unit ball graph induced by $P$, denoted $U B G(P)$, is defined analogously.

Given $d \in[0,1]$, graph $G=(V, E)$ can be realized as a $d$-quasi unit disc graph, denoted $d$-QUDG, if there exists an embedding of $G, f: V \rightarrow \mathbb{R}^{2}$, such that for all $u, v \in V$, 
1. $\|f(u)-f(v)\| \leq d \Rightarrow(u, v) \in E$, and

2. $\|f(u)-f(v)\|>1 \Rightarrow(u, v) \notin E$.

If $\|f(u)-f(v)\| \in(d, 1]$, then no conclusion may be drawn about the membership of edge $(u, v)$ in $E$ : both $(u, v) \in E$ and $(u, v) \notin E$ are possible. Observe that a 1-QUDG is a UDG and any graph is a 0-QUDG. See Barrière et al. [6] and Kuhn et al. [23] for a discussion of quasi unit disc graphs.

Given a fixed $d$, let $\mathcal{U D G}, \mathcal{U B G}$, and $d-\mathcal{U} B \mathcal{B}$ denote the classes of graphs that can be realized as a UDG, a UBG, or a $d$-QUDG, respectively.

Finally, if $P_{1}$ and $P_{2}$ denote parallel planes in $\mathbb{R}^{3}$, we refer to the closed region between $P_{1}$ and $P_{2}$ as a slab and to the minimum distance between $P_{1}$ and $P_{2}$ as its thickness.

\section{Routing in Unit Ball Graphs}

In this section we present our main results on routing in unit ball graphs in Theorems 1 and 2. Together, these two results characterize the class of UBGs for which a $k$-local routing algorithm is possible. Our first observation, stated formally in the following lemma, is that any UBG contained in a slab of thickness $\lambda<1$ can be transformed into a QUDG by projecting the points in the UBG to a plane parallel to the slab.

Lemma 1 Choose any $\lambda \leq 1$ and let $P$ denote $a$ set of points in $\mathbb{R}^{3}$ contained in a slab of thickness $\lambda$. Let $f: \mathbb{R}^{3} \rightarrow \mathbb{R}^{2}$ denote the projection onto a plane parallel to the slab. Let $G=(V, E)$ denote the embedded graph such that $V=\{f(v) \mid v \in P\}$ and $E=\{(f(u), f(v)) \mid\|u-v\| \leq 1, u, v \in P\} \quad(V$ and $E$ may be multisets). $G$ is a $\left(\sqrt{1-\lambda^{2}}\right)-Q U D G$.

Proof. Choose any two points $u, v \in P$. If $\|f(u)-f(v)\|>1$ then $\|u-v\|>1$ and $(f(u), f(v)) \notin E$. Similarly, if $\|f(u)-f(v)\| \leq$ $\sqrt{1-\lambda^{2}}$ then $\|u-v\| \leq 1$ and $(f(u), f(v)) \in E$. Therefore, the projected graph $G$ is a $\left(\sqrt{1-\lambda^{2}}\right)$ quasi unit disc graph.

Kuhn et al. [23] propose a 2-local routing algorithm for $d$-quasi unit disc graphs that succeeds for any $d \geq 1 / \sqrt{2}$. The following theorem is an immediate consequence of Lemma 1.

Theorem 1 There exists a 2-local routing algorithm that succeeds for the class of graphs $\mathcal{G}=\{U B G(P) \mid$ $P \subseteq \mathbb{R}^{3}$ is contained in a slab of thickness $\left.1 / \sqrt{2}\right\}$.
Proof. By Lemma 1, the projection of $U B G(P)$ onto a plane parallel to the slab is a $1 / \sqrt{2}$-QUDG, $G$. Since $U B G(P)$ and $G$ are isomorphic, the $k$ neighbourhood of a vertex $v$ in $U B G(P)$ determines the $k$-neighbourhood of the corresponding vertex in $G$. Therefore, a 2-local routing algorithm in $U B G(P)$ can be achieved by projecting the 2-neighbourhood of the current vertex $v$ and simulating a 2-local routing algorithm such as the one in [23] on the corresponding QUDG.

Note that Theorem 1 requires knowledge of a normal to the plane since, in general, this cannot be determined from the 2-neighbourhood of a vertex.

In the remainder of this section, we show that the result in Theorem 1 is tight: there is no $k$-local routing algorithm that can guarantee delivery on all UBGs contained in slabs thicker than $1 / \sqrt{2}$. To prove this, we first show that any such algorithm would imply the existence of a 1-local routing algorithm for arbitrary graphs (Lemma 2). Next we show the impossibility of a 1-local routing algorithm for arbitrary labelled graphs (Lemma 3).

Lemma 2 If there exists some $\epsilon>0$, some $k \geq$ 1 , and a k-local routing algorithm that succeeds for $U B G(P)$, for every finite set of points $P$ in $\mathbb{R}^{3}$ contained within a slab of thickness $1 / \sqrt{2}+\epsilon$, then there exists a 1-local routing algorithm that succeeds for any connected, labelled graph $G$.

Proof. Suppose there exists an $\epsilon>0$ and a $k$-local algorithm $\mathcal{A}$ that succeeds in routing on every UBG contained in a slab of thickness $1 / \sqrt{2}+\epsilon$. For any arbitrary graph $G$, we show how to construct a UBG $G^{\prime}$ such that routing on $G$ can be accomplished by simulating $\mathcal{A}$ on $G^{\prime}$. Let $G=(V, E)$ be an arbitrary connected labelled graph. Let $n=|V|$. Without loss of generality, assume the vertices are labelled $0, \ldots, n-1$; that is, $V=\{0, \ldots, n-1\}$. The proof holds regardless of whether the set of vertex labels is a contiguous subset of the integers.

We define a transformation from $G$ to a set of points $P(G)$ in $\mathbb{R}^{3}$. Let $\epsilon^{\prime}=\min \{\epsilon, \sqrt{3}-1 / 2\}$. For each vertex $v \in V$, create two sets $C_{v}=$ $\{(2 v k, y \pm 1 / 2,0) \mid y \in\{2 k \cdot \min (N(v))-(k-$ $1), \ldots, 2 k \cdot \max (N(v))+(k-1)\}\}$ and $R_{v}=\{(x \pm$ $\left.1 / 2,2 v k, 1 / \sqrt{2}+\epsilon^{\prime}\right) \mid x \in\{2 k \cdot \min (N(v))-(k-$ $1), \ldots, 2 k \cdot \max (N(v))+(k-1)\}\}$, where $N(v)$ denotes the set of labels of neighbours of $v$ and $v$ itself. That is, $C_{v}$ is a column of points in the $x y$ plane starting at $(2 v k, 2 k \min (N(v))-k+1 / 2,0)$ and 


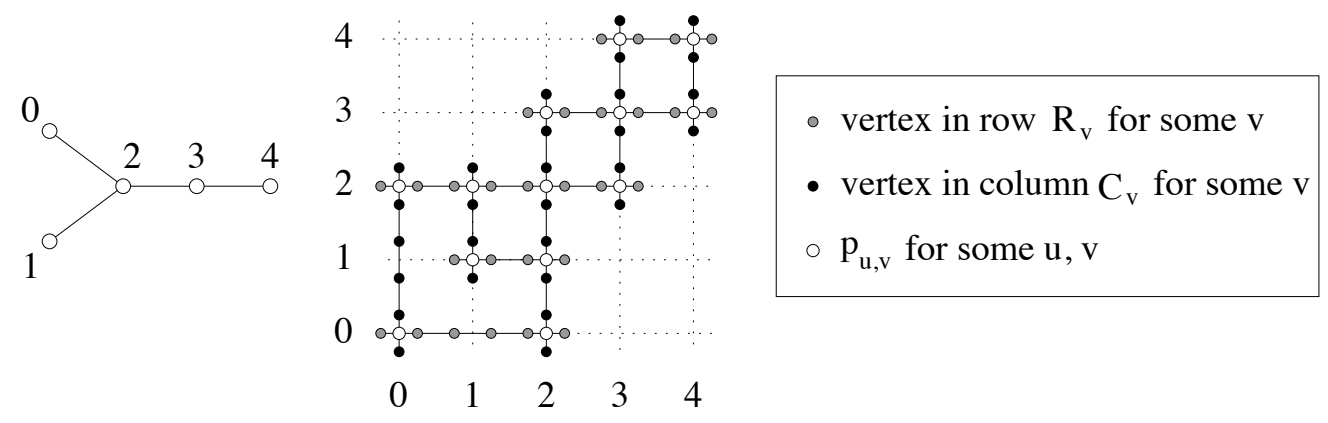

Figure 1: A graph $G$ and the corresponding graph $U B G(P(G))$ for $k=1$
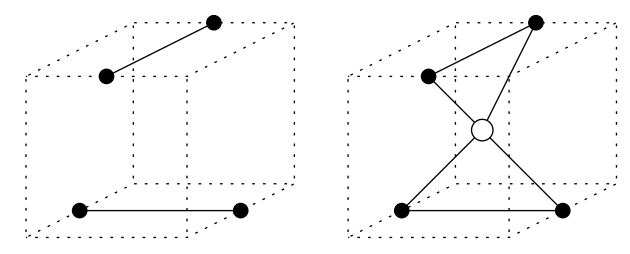

Figure 2: The region $[2 i k \pm 1 / 2] \times[2 j k \pm 1 / 2] \times$ $\left[0,1 / \sqrt{2}+\epsilon^{\prime}\right]$ in $U B G(P(G))$ if $i$ and $j$ are not adjacent in $G$, and the same region if $i$ and $j$ are adjacent in $G$

$R_{v}$ is a row of points in the $x y$-plane starting at $\left(2 k \min (N(v))-k+1 / 2,2 v k, 1 / \sqrt{2}+\epsilon^{\prime}\right)$. For each edge $(u, v) \in E$, add a point $p_{u, v}=(2 u k, 2 v k,(1 / \sqrt{2}+$ $\left.\epsilon^{\prime}\right) / 2$ ). Finally, for each $v \in V$, add the point $p_{v, v}=$ $\left(2 v k, 2 v k,\left(1 / \sqrt{2}+\epsilon^{\prime}\right) / 2\right)$. The graph $U B G(P(G))$ is defined in the usual way; every pair of points within distance one of each other is connected by an edge. Figure 1 shows a graph $G$ and the corresponding graph $U B G(P(G))$ for $k=1$.

For each $v \in V$, the set $C_{v}$ (similarly, $R_{v}$ ) is a sequence of points, each at distance one from the previous point, and therefore, $C_{v}\left(R_{v}\right)$ corresponds to a path in $U B G(P(G))$. For any $u \neq v$, columns $C_{u}$ and $C_{v}$ are at distance at least two apart and rows $R_{u}$ and $R_{v}$ are at distance at least two apart. If edge $(u, v) \notin E$, where $u \neq v$, then the distance between any point $i \in C_{u}$ and any point $j \in R_{v}$ is greater than one; therefore, $i$ and $j$ are not adjacent in $U B G(P(G))$. Since $\epsilon^{\prime} \leq \sqrt{3}-1 / \sqrt{2}$, if edge $(u, v) \in E$, then the distance between some point $i \in R_{u}$ and $p_{u, v}$ is at most one and the distance between some point $j \in C_{v}$ and $p_{u, v}$ is at most one; therefore, $i$ and $p_{i, j}$ are adjacent in $U B G(P(G))$, as are $j$ and $p_{i, j}$. See Figure 2 .

It is straightforward to see that $U B G(P(G))$ is contained within a slab of thickness $1 / \sqrt{2}+\epsilon^{\prime} \leq 1 / \sqrt{2}+\epsilon$ and therefore algorithm $\mathcal{A}$ should succeed on it. We claim that a straightforward simulation of $\mathcal{A}$ in $U B G(P(G))$ constitutes a 1-local routing algorithm for $G$. That is, upon reaching a vertex $v \in V$, it suffices to simulate $\mathcal{A}$ on the subgraph of $U B G(P(G))$ that corresponds to vertex $v$ and its 1 neighbourhood in $G$. The simulation begins at point $p_{v, v}$ with the goal of reaching the destination vertex $p_{t, t}$. When the simulation moves to a point outside $C_{v} \cup R_{v} \cup\left\{p_{v, v}\right\}$ in $U B G(P(G))$, it must reach a point $p_{v, u}$ or $p_{u, v}$ for some $u \neq v$. This corresponds to forwarding the message to vertex $u$, which must be a neighbour of $v$ in $G$. The computation of the $k$-local subgraph of $U B G(P(G))$ around any vertex in $C_{v} \cup R_{v} \cup\left\{p_{v, v}\right\}$, and hence the simulation, can be performed completely locally for any vertex $v$, given the 1-neighbourhood of $v$ in $G$. Furthermore, knowledge of the number of vertices in $G$ is not required to simulate the local neighbourhood of $v$ in $U B G(P(G))$. Since the simulation results in a 1-local routing algorithm guaranteed to succeed on an arbitrary graph $G$, the lemma follows.

We proceed to show that no 1-local routing algorithm succeeds on all labelled graphs.

Lemma 3 For any 1-local routing algorithm $\mathcal{A}$, there exists a connected labelled graph for which $\mathcal{A}$ is defeated.

Proof. Suppose $\mathcal{A}$ is any 1-local routing algorithm defined on all unembedded labelled graphs for some set of vertex label $\Sigma$ and let $f$ denote the corresponding routing function. Function $f$ must be defined for all valid combinations of input. In particular, $f\left(s, t, v_{i}, u,\left\{\left(u, v_{1}\right), \ldots,\left(u, v_{j}\right)\right\}\right)$ must be defined for all $i \in\{1, \ldots, j\}$, where $s$ denotes the origin, $t$ denotes the destination, $v_{i}$ denotes the last vertex visited, $u$ denotes the current vertex, and 

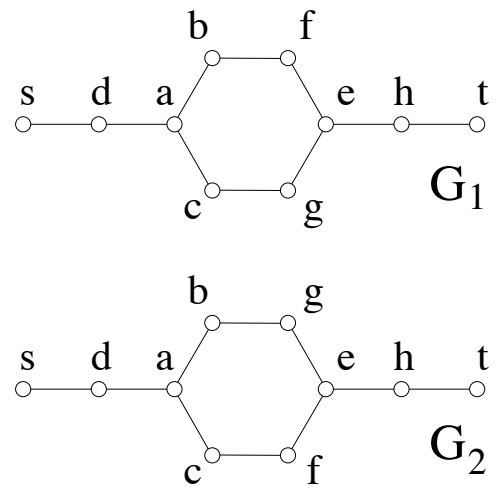

Figure 3: Any routing algorithm is defeated by $G_{1}$ or $G_{2}$ if all local routing functions are derangements.

$\left\{v_{1}, \ldots, v_{j}\right\}$ denotes the set of neighbours of $u$. Let $f_{u}^{\prime}\left(v_{i}\right)=f\left(s, t, v_{i}, u,\left\{\left(u, v_{1}\right), \ldots,\left(u, v_{j}\right)\right\}\right)$ for a given $s, t, u$, and its set of neighbours. We refer to $f_{u}^{\prime}$ as a local routing function.

Consider the properties of $f_{u}^{\prime}$ when the 1neighbourhood of $u$ is a tree. Function $f_{u}^{\prime}$ : $\left\{v_{1}, \ldots, v_{j}\right\} \rightarrow\left\{v_{1}, \ldots, v_{j}\right\}$ is one of $j^{j}$ possible functions. Function $f_{u}^{\prime}$ must be bijective. Suppose otherwise. Without loss of generality, say $f_{u}^{\prime}\left(v_{i}\right) \neq v_{1}$ for all $i \in\{1, \ldots, j\}$. Function $f_{u}^{\prime}$ is defeated by a tree with $t$ in the subtree of $u$ rooted at $v_{1}$ and $s$ in any other subtree of $u$. Furthermore, if $j>1$ then $f_{u}^{\prime}$ must be a derangement. Suppose instead that $f_{u}^{\prime}\left(v_{i}\right)=v_{i}$ for some $i \in\{1, \ldots, j\}$. Function $f_{u}^{\prime}$ is defeated by a tree with $s$ in the subtree of $u$ rooted at $v_{i}$ and $t$ in any other subtree of $u$. Therefore, it suffices to consider local routing functions $f_{u}^{\prime}$ that are derangements.

A set of cardinality two has a unique derangement. Therefore, $f_{u}^{\prime}$ is uniquely defined when $u$ has degree two. A set of cardinality three has two possible derangements. Therefore, $f_{u}^{\prime}$ is one of two functions when $u$ has degree two. Observe that $f_{u}^{\prime}$ is also uniquely defined when $u$ has degree one.

Let $G_{1}$ and $G_{2}$ denote the graphs illustrated in Figure 3. Graphs $G_{1}$ are $G_{2}$ are automorphic upon permuting vertices $f$ and $g$. Both $G_{1}$ and $G_{2}$ are triangle-free; that is, the 1-neighbourhood of every vertex is a tree. As discussed, the local routing function is uniquely defined for all vertices of degree two or less. There are two vertices of degree three: $a$ and $e$. Let $f_{a}^{\prime}(v)=f(s, t, v, a,\{(a, b),(a, c),(a, d)\})$ and $f_{e}^{\prime}(v)=f(s, t, v, e,\{(e, f),(e, g),(e, h)\})$ denote the local routing functions for vertices $a$ and $e$, respectively. Each of $f_{a}^{\prime}$ and $f_{e}^{\prime}$ may be defined by routing function 1

\begin{tabular}{|c|c|}
\hline$u$ & $f_{a}^{\prime}(u)$ \\
\hline$b$ & $c$ \\
$c$ & $d$ \\
$d$ & $b$ \\
\hline
\end{tabular} \mid \begin{tabular}{|c|c|}
\hline$u$ & $f_{e}^{\prime}(u)$ \\
\hline$f$ & $g$ \\
$g$ & $h$ \\
$h$ & $f$ \\
\hline
\end{tabular}

\begin{tabular}{|c|c|}
\hline$u$ & $f_{a}^{\prime}(u)$ \\
\hline$b$ & $d$ \\
$c$ & $b$ \\
$d$ & $c$ \\
\hline
\end{tabular} \mid \begin{tabular}{|c|c|}
\hline$u$ & $f_{e}^{\prime}(u)$ \\
\hline$f$ & $g$ \\
$g$ & $h$ \\
$h$ & $f$ \\
\hline
\end{tabular}

routing function 3

\begin{tabular}{|c|c|}
\hline$u$ & $f_{a}^{\prime}(u)$ \\
\hline$b$ & $c$ \\
$c$ & $d$ \\
$d$ & $b$ \\
\hline
\end{tabular}

\begin{tabular}{|c|c|}
\hline$u$ & $f_{e}^{\prime}(u)$ \\
\hline$f$ & $h$ \\
$g$ & $f$ \\
$h$ & $g$ \\
\hline
\end{tabular}

routing function 4

\begin{tabular}{|c|c|}
\hline$u$ & $f_{a}^{\prime}(u)$ \\
\hline$b$ & $d$ \\
$c$ & $b$ \\
$d$ & $c$ \\
\hline
\end{tabular} \mid \begin{tabular}{|c|c|}
\hline$u$ & $f_{e}^{\prime}(u)$ \\
\hline$f$ & $h$ \\
$g$ & $f$ \\
$h$ & $g$ \\
\hline
\end{tabular}

Table 1: The four combinations of derangements for local routing functions $f_{a}^{\prime}$ and $f_{e}^{\prime}$

one of two derangements, resulting in four possible routing functions for graphs $G_{1}$ and $G_{2}$, given in Table 1. As shown in Figure 4, each of the four routing functions is defeated by either $G_{1}$ or $G_{2}$.

Lemma 4 For any fixed $k \geq 1$ and any $k$-local routing algorithm $\mathcal{A}$, there exists a labelled graph for which $\mathcal{A}$ is defeated.

Proof. The result follows by an argument analogous to the proof of Lemma 3, upon replacing the edges in graphs $G_{1}$ and $G_{2}$ by paths of length $k$.

Furthermore, by replacing the edges with paths of length $\max (k, l)$, it is straightforward to generalize Lemma 4 such that it remains true even if a routing algorithm has knowledge of the previous $l$ nodes on the route, for any fixed $l \geq 1$. In this case, a routing function has the form $f: \Sigma^{2} \times \Sigma^{l} \times \Sigma \times \mathscr{P}\left(\Sigma^{2}\right) \rightarrow \Sigma$.

The following theorem is an immediate consequence of Lemmas 2 and 4.

Theorem 2 For every $\epsilon>0$, every $k \geq 1$, and every $k$-local routing algorithm $\mathcal{A}$, there exists a finite set of points $P$ in $\mathbb{R}^{3}$ contained within a slab of thickness $1 / \sqrt{2}+\epsilon$ such that $\mathcal{A}$ is defeated by $U B G(P)$.

Theorem 2 and Lemma 1 also give the following corollary:

Corollary 1 For every $\epsilon \in(0,1 / \sqrt{2}]$, every $k \geq 1$, and every $k$-local routing algorithm $\mathcal{A}$, there exists a $(1 / \sqrt{2}-\epsilon)-Q U D G, G$, such that $\mathcal{A}$ is defeated by $G$.

In [6] and [23], algorithms for routing in $d$-QUDGs for $d \geq 1 / \sqrt{2}$ are given. Corollary 1 implies that these results are tight: it is impossible to extend the range of $d$ for which the class of $d$-QUDGs would admit a $k$-local algorithm. 


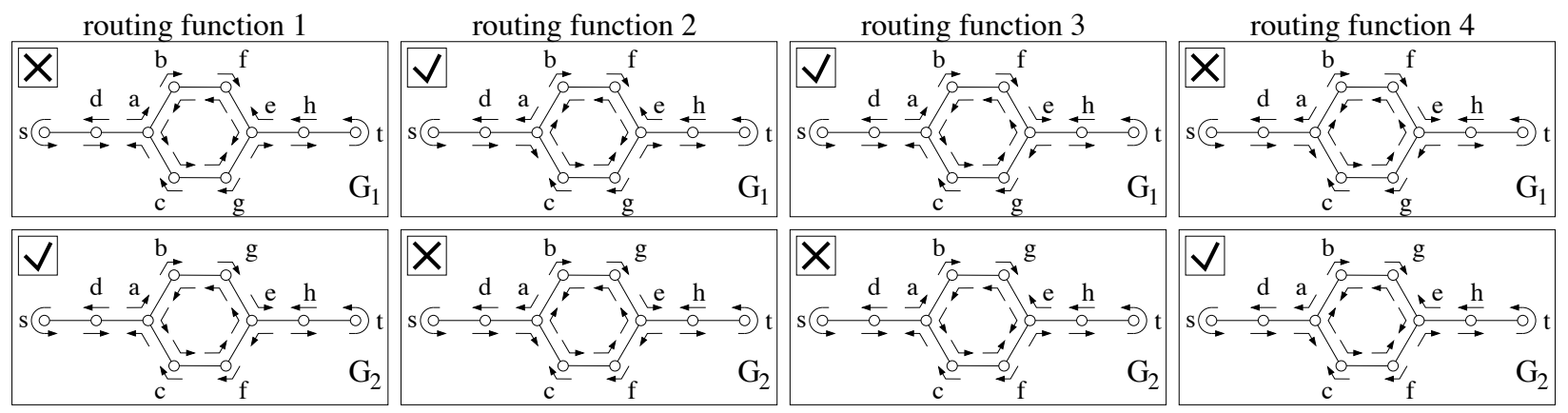

Figure 4: Four routing functions are possible for graphs $G_{1}$ and $G_{2}$ such that each local routing function is a derangement. Each routing function is defeated by $G_{1}$ or $G_{2}$ when delivering a message from $s$ to $t$. A defeat is denoted by $\mathrm{X}$.

\section{Unit Ball Graphs and Quasi Unit Disc Graphs}

In Section 3 we showed that any UBG contained within a slab of thickness $\lambda \leq 1$ is isomorphic to some $\left(\sqrt{1-\lambda^{2}}\right)$-QUDG. In this section we present additional observations on unit ball graphs and their relationship to quasi unit disc graphs and more general graphs. We show the following general result which follows from Lemmas 5 and 6 .

Theorem 3 (1) Given any fixed $d>0, \mathcal{U B G} \nsubseteq d$ $\mathcal{Q U D G}$. (2) Given any fixed $d^{\prime}<\sqrt{3} / 2, d^{\prime}-\mathcal{Q U D} \mathcal{G} \nsubseteq$ $\mathcal{U B G}$.

We first show that the class $\mathcal{U B G}$ is not contained within the class $d-\mathcal{Q U D G}$ for any fixed $d$ :

Lemma 5 For every $d>0$, there exists a finite set of points $P$ in $\mathbb{R}^{3}$ such that $U B G(P)$ is not isomorphic to any $d-Q U D G$.

Proof. Choose any $d \in(0,1]$ and any integer $k>$ $5 / d^{2}$. Let $P=\mathbb{Z}^{3} \cap[-k, k]^{3}$. Set $P$ is contained within a slab of thickness $2 k+1$ and has cardinality $(2 k+$ $1)^{3}$. $U B G(P)$ is the grid graph $G_{2 k+1,2 k+1,2 k+1}$. Let $P^{\prime}$ denote the subset of $P$ consisting of alternating vertices in the grid such that $P^{\prime}$ does not include the origin, i.e., $\left(p_{x}, p_{y}, p_{z}\right) \in P^{\prime}$ if and only if $p \in P$ and $p_{x}+p_{y}+p_{z}$ is odd. Any two vertices in $P^{\prime}$ are a distance greater than one apart. Consequently, $P^{\prime}$ is an independent set of $U B G(P)$ with cardinality $\lceil|P| / 2\rceil$.

For any vertex $u \in P$, the graph distance between $u$ and the origin in $U B G(P)$ is at most $3 k$. Consequently, any realization of $U B G(P)$ as a $d$-quasi unit disc graph must be contained within a disc of radius
$3 k$. Furthermore, all vertices of $P^{\prime}$ must be a distance greater than $d$ apart from each other. Consequently, $\left|P^{\prime}\right|$ non-intersecting discs of radius $d / 2$ must be contained within a disc of radius $3 k+d / 2$. Summing the areas of the $\left|P^{\prime}\right|$ discs gives the following constraint:

$$
\pi\left(\frac{d}{2}\right)^{2}\left\lceil\frac{(2 k+1)^{3}}{2}\right\rceil<\pi\left(3 k+\frac{d}{2}\right)^{2} .
$$

It is straightforward to show that (1) is false for any $k>5 / d^{2}$. Therefore, $G_{2 k+1,2 k+1,2 k+1}$ cannot be realized as a $d$-QUDG.

If $d$ is not fixed, however, then any graph can be realized as a $d$-QUDG for some $d$ :

Proposition 1 For every finite labelled graph $G$, there exists a $d>0$ and a $d-Q U D G, G^{\prime}$ such that $G$ is isomorphic to $G^{\prime}$.

Proof. Choose any graph $G$. Embed all vertices of $G$ at distinct points in a disc of radius $1 / 2$ in the plane. Add the edges of $G$. Choose $d>0$ such that $d$ is less than the minimum distance between any two points. The resulting graph is a $d$-QUDG since all edges have lengths in the range $[d, 1]$.

By Lemma 1, any UBG contained in a slab of thickness $\lambda<1$ is isomorphic to some quasi unit disc graph. The converse is not true; as we show in Lemma 6, there exist quasi unit disc graphs that are not isomorphic to any UBG.

Lemma $6 K_{3,3}$ is forbidden as an induced subgraph of a $U B G$ but can be realized as a $(\sqrt{3} / 2-\epsilon)-Q U D G$ for any $\epsilon>0$. 

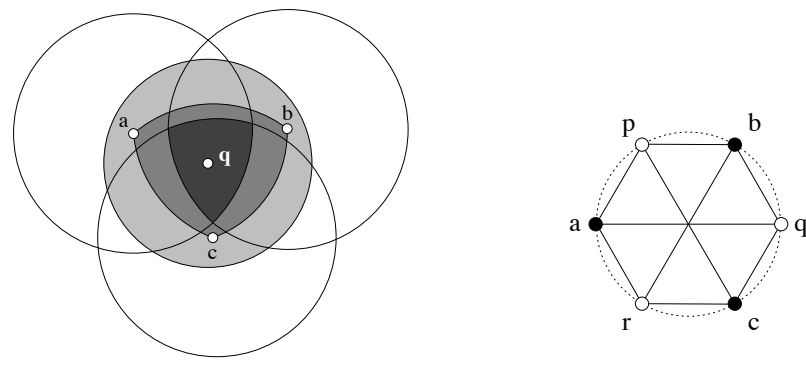

Figure 5: Illustrations in support of Lemma 6. left: $R \cap H \subseteq 1-H(\{a, b, c\}) \subseteq D$. right: A realization of $K_{3,3}$ as a $(\sqrt{3} / 2-\epsilon)$-QUDG for any $\epsilon>0$.

Proof. Let $\{a, b, c\}$ and $\{p, q, r\}$ denote sets of points in $\mathbb{R}^{3}$ that correspond to the two partitions of $K_{3,3}$. The distance between any two points in a partition must be greater than one in any realization of $K_{3,3}$ as a UBG. Let $R$ denote the intersection of three unit balls with respective centres $a, b$, and $c$. Assume $R$ is non-empty, since $p, q$, and $r$ must be contained in $R$. Let $H$ denote the plane through points $a, b$, and $c$. Let $H^{+}$and $H^{-}$denote the closed half-spaces induced by $H$. Let $R^{+}=H^{+} \cap R$ and $R^{-}=H^{-} \cap$ $R$. At least two points of $p, q$, and $r$ must both lie in $R^{+}$or $R^{-}$. Without loss of generality, assume $\{p, q\} \subseteq R^{+}$. We derive a contradiction by showing that $\|p-q\| \leq 1$.

Observe that the projection of $p$ and $q$ onto $H$ remains in $R^{+}$. Therefore $\|p-q\|$ is maximized when at least one of $p$ or $q$ lies on $H$. Without loss of generality, assume $q$ lies on $H$. Since $p \in R$, a unit ball centred at $p$ must contain $a, b$, and $c$. Let $D$ denote the disc defined by the intersection of this unit ball with $H$. The boundary of $R \cap H$ consists of three arcs of unit circles. Observe that $R \cap H$ must be contained within the 1-hull of $\{a, b, c\}$, denoted $1-H(\{a, b, c\})$ (i.e., the intersection of all unit discs that contain $\{a, b, c\})$. See Figure 5. Since the radius of $D$ is at most one and $\{a, b, c\} \in D$, it follows that $q \in R \cap H \subseteq 1-H(\{a, b, c\}) \subseteq D$. Since $q \in D$, $q$ is contained within the unit ball centred at $p$ and, therefore, $\|p-q\| \leq 1$.

$K_{3,3}$ can be realized as a $(\sqrt{3} / 2-\epsilon)$-quasi unit disc graph for any $\epsilon>0$ by positioning vertices in alternating partitions uniformly on a circle of radius 1/2. See Figure 5.

Therefore, $d-\mathcal{Q U D G} \subseteq \mathcal{U B G}$ when $d=1$ but $d$ $\mathcal{Q U D G} \nsubseteq \mathbb{U} B \mathcal{G}$ when $d \leq \sqrt{3} / 2$. It remains open to determine for which range of values of $d \in(\sqrt{3} / 2,1)$ the predicate $d-\mathcal{Q U D G} \subseteq \mathcal{U B G}$ remains true.

The definition of a $d$-quasi unit disc graph has a natural generalization to three dimensions as a $d$ quasi unit ball graph, denoted $d$-QUBG. We note the following straightforward relationship between quasi unit ball graphs and quasi unit disc graphs:

Proposition 2 For every $d \leq 1$, every $\lambda<d$, and every d-QUBG, $G$, contained in a slab of thickness $\lambda$, there exists a $\left(\sqrt{d^{2}-\lambda^{2}}\right)-Q U D G G^{\prime}$ such that $G$ is isomorphic to $G^{\prime}$.

Proof. The result follows by an argument analogous to the proof of Lemma 1.

Proposition 2 and the 2-local routing algorithm of Kuhn et al. [23] give:

Corollary 2 There exists a 2-local routing algorithm that succeeds for any $d$-QUBG, $G$, such that $d \geq$ $\sqrt{\lambda^{2}+1 / 2}$ and $G$ is contained in a slab of thickness $\lambda$.

Finally, we show that any independent set of the set of neighbours of any vertex in a UBG has cardinality at most 12. That is, $K_{1,12}$ can be realized as an induced subgraph of a UBG but $K_{1,13}$ cannot.

Proposition 3 There are no induced stars with degree greater than 12 in any unit ball graph.

Proof. The kissing number in dimension $d$ is defined to be $k$ if $k$ is the maximum number of $d$-spheres of radius $r$ that can be packed around a central $d$-sphere of radius $r$ such that each $d$-sphere kisses the central $d$-sphere (i.e., the boundaries of the two $d$-spheres intersect in a point) and the intersection of the interiors of any two $d$-spheres is empty. The kissing number in three dimensions is 12 [13]. Furthermore, realizations exist such that twelve spheres kiss a thirteenth sphere and all pairwise intersections for the first twelve spheres are empty [13] (e.g., the spheres kiss at the vertices of a regular icosahedron). It follows that for $r=1 / 2$, such an embedding gives a realization of $K_{1,12}$ as a UBG.

Suppose there exists an embedding of $K_{1,13}$ as a UBG. Let $V \subseteq \mathbb{R}^{3}$ denote the vertex set of such a graph $G$. Observe that no two vertices in $V$ may coincide. Let $c$ denote the vertex of degree thirteen in $V$ and let $u$ and $v$ denote two different vertices in $V-\{c\}$. Let $u^{\prime}$ denote the point that lies at unit distance from $c$ on the ray $\overrightarrow{c u}$. Since $\|u-c\| \leq 1,\|v-c\| \leq 1$, and $\|u-v\|>1$, therefore, 
$\angle u c v>2 \pi / 3$. Consequently, $\left\|u^{\prime}-v\right\| \geq\|u-v\|$. That is, each vertex $w$ in $V-\{c\}$ can be moved to unit distance from $c$ along the ray $\overrightarrow{c w}$ without altering adjacencies in the corresponding UBG. Centring a sphere of radius $1 / 2$ at each of the resulting vertices gives thirteen mutually non-intersecting spheres kissing a fourteenth central sphere. This derives a contradiction; our assumption must be false and no such graph $G$ can exist.

In $\mathbb{R}^{3}$, the bound from Proposition 3 matches the kissing number. In $\mathbb{R}^{2}$, the cardinality of the independent set of the neighbours of a vertex in a UDG is strictly less than the kissing number: a unit disc graph has no induced stars with degree greater than 5 [11] whereas the kissing number for unit circles is 6 [13].

\section{Discussion}

We have shown the impossibility of routing algorithms that guarantee delivery in three-dimensional ad hoc networks, modelled by unit ball graphs, when nodes are constrained to have information only about their $k$-hop neighbourhood. This result is in direct contrast to the two-dimensional case, where a 1-local algorithm such as face routing guarantees delivery on all unit disc graphs.

The results from the planar case do "lift off" the plane to a limited extent. We showed that unit ball graphs for which the nodes are contained in a slab of thickness $1 / \sqrt{2}$ admit a 2-local routing algorithm that guarantees delivery. On the other hand, we also showed that for any fixed $k$, there is no $k$-local routing algorithm that is guaranteed to succeed on all unit ball graphs, even if the nodes are contained in a slab of thickness of $1 / \sqrt{2}+\epsilon$ for arbitrarily small $\epsilon>0$. An interesting question would be to characterize precisely the class of unit ball graphs in thicker slabs that do have routing algorithms. Since distributed algorithms for routing in unit ball graphs remain an urgent necessity, the question of the kind of information with which a routing algorithm might be augmented in order to circumvent the negative results in this paper would be useful to answer. Shortly before submitting the final version of this manuscript, the authors learned of recent results helping achieve this goal, including using randomization [16] and allowing nodes to read and write to a small number of additional bits passed along with the message [10].

In this paper, we have begun an exploration of the relationship between unit ball graphs, quasi unit disc graphs, and quasi unit ball graphs. Many questions remain open. For example: does there exist a $\delta>0$ such that any $(1-\delta)$-QUDG is isomorphic to some UBG? If so, what is the supremum of all such $\delta$ ? By Lemma $6, \delta<1-\sqrt{3} / 2$. Several graph problems that are NP-complete are efficiently approximable (e.g., maximum independent set, graph coloring, and minimum dominating set [27]) or tractable (e.g., maxclique [12]) on unit disc graphs. A similar investigation of which graph problems are tractable or approximable on unit ball graphs and the other classes of graphs studied here might be a fruitful avenue of research.

\section{Acknowledgements}

The authors would like to thank Prosenjit Bose for providing helpful answers to our questions regarding hardness results for memoryless routing algorithms as well as Stephen Wismath, Ethan Kim, and John Iacono for discussing ideas which resulted in a preliminary algorithm for routing in unit ball graphs at the 2007 Bellairs Workshop on Computational Geometry.

\section{References}

[1] A. Abdallah, T. Fevens, and J. Opatrny. Hybrid position-based 3-D routing algorithms with partial flooding. In Proceedings of the IEEE Canadian Conference on Electrical and Computer Engineering, 2006.

[2] A. Abdallah, T. Fevens, and J. Opatrny. Randomized 3-D position-based routing algorithms for ad-hoc networks. In Proceedings of the Conference on Mobile and Ubiquitous Systems, 2006.

[3] A. Abdallah, T. Fevens, and J. Opatrny. Poweraware 3D position-based routing. In Proceedings of the IEEE International Conference on Communications, pages 3130-3135, 2007.

[4] I. F. Akyildiz, D. Pompili, and T. Melodia. Underwater acoustic sensor networks: Research challenges. Ad Hoc Networks, 2005.

[5] S. M. N. Alam and Z. J. Haas. Coverage and connectivity in three-dimensional networks. In Proceedings of the Conference on Mobile Computing and Networking, pages 346-357, 2006. 
[6] L. Barrière, P. Fraigniaud, L. Narayanan, and J. Opatrny. Robust position-based routing in wireless ad hoc networks with unstable transmission ranges. Wireless Communications and Mobile Computing, 3/2:141-153, 2003. extended abstract appears in Proceedings of the Workshop on Discrete Algorithms and Methods for Mobile Computing and Communications, 2000.

[7] P. Bose, A. Brodnik, S. Carlsson, E. D. Demaine, R. Fleischer, A. López-Ortiz, P. Morin, and I. Munro. Online routing in convex subdivisions. International Journal of Computational Geometry and Applications, 12(4):283-295, 2002.

[8] P. Bose and P. Morin. Online routing in triangulations. In Proceedings of International Symposium on Algorithms and Computation, volume 1741 of Lecture Notes in Computer Science, pages 113-122. Springer-Verlag, 1999.

[9] P. Bose, P. Morin, I. Stojmenovic, and J. Urrutia. Routing with guaranteed delivery in ad hoc wireless networks. Wireless Networks, 7:609616, 2001.

[10] M. Braverman. On ad hoc routing with guaranteed delivery, 2008. arXiv:0804.0862v1.

[11] H. Breu. Algorithmic Aspects of Constrained Unit Disk Graphs. $\mathrm{PhD}$ thesis, University of British Columbia, 1996.

[12] B. Clark, C. Colbourn, and D. Johnson. Unit disk graphs. Discrete Mathematics, 86(1-3):165177, 1990 .

[13] J. H. Conway and N. J. A. Sleane. Sphere packings and kissing numbers. In J. H. Conway and N. J. A. Sleane, editors, Sphere Packings, Lattices and Groups, pages 1-30. Springer, third edition, 1999.

[14] S. Durocher, D. Kirkpatrick, and L. Narayanan. On routing with guaranteed delivery in threedimensional ad hoc wireless networks. In Proceedings of the International Conference on Distributed Computing and Networking, volume 4904 of Lecture Notes in Computer Science, pages 546-557. Springer-Verlag, 2008.

[15] T. Fevens, G. Kao, and J. Opatrny. 3-D localized position-based routing with nearly certain delivery in mobile ad hoc networks. In Proceedings of the International Symposium on Wireless Pervasive Computing, 2007.
[16] R. Flury and R. Wattenhofer. Randomized 3D geographic routing. In Proceedings of the IEEE Conference on Computer Communications, volume 27, pages 834-842, 2008.

[17] S. Giordano and I. Stojmenovic. Position based routing algorithms for ad hoc networks: A taxonomy. In X. H. X. Cheng and D.-Z. Du, editors, Ad Hoc Wireless Networking, pages 103136. Kluwer, 2003.

[18] J. Heidemann, W. Ye, J. Wills, A. Syed, and Y. Li. Research challenges and applications for underwater sensor networking. In Proceedings of the IEEE Wireless Communications and Networking Conference, 2006.

[19] S. Kamali and J. Opatrny. Posant: a position based ant colony routing algorithm for mobile ad-hoc networks. In Proceedings of International Conference on Wireless and Mobile Communications, volume 3, 2007.

[20] B. Karp and H. Kung. GPSR: Greedy perimeter stateless routing for wireless networks. In Proceedings of the Conference on Mobile Computing and Networking, volume 6, pages 243-254, 2000.

[21] E. Kranakis, H. Singh, and J. Urrutia. Compass routing on geometric networks. In Proceedings of the Canadian Conference on Computational Geometry, volume 11, pages 51-54, 1999.

[22] F. Kuhn, R. Wattenhofer, and A. Zollinger. Asymptotically optimal geometric mobile adhoc routing. In Proceedings of the International Workshop on Discrete Algorithms and Methods for Mobile Computing and Communications, volume 6, pages 24-33, 2002.

[23] F. Kuhn, R. Wattenhofer, and A. Zollinger. Adhoc networks beyond unit disk graphs. In Proceedings of the ACM Joint Workshop on Foundations of Mobile Computing, 2003.

[24] X.-Y. Li. Applications of computational geometry in wireless ad hoc networks. In X. Cheng, X. Huang, and D.-Z. Du, editors, Ad Hoc Wireless Networking. Kluwer, 2003.

[25] X.-Y. Li and Y. Wang. Wireless sensor networks and computational geometry. In M. I. et al., editor, Handbook of Sensor Networks. CRC Press, 2003. 
[26] X. Lin and I. Stojmenović. GEDIR: Loopfree location based routing in wireless networks. In Proceedings of the Conference on Parallel and Distributed Computing and Systems, pages 1025-1028, 1999.

[27] M. V. Marathe, H. Breu, H. Hunt III, S. S. Ravi, and D. J. Rosenkrantz. Simple heuristics for unit disk graphs. Networks, 25:59-68, 1995.

[28] D. Peleg. Distributed computing: a localitysensitive approach. SIAM, 2000.

[29] C. Perkins. Ad Hoc Networking. Addison-Wesley Professional, 2001.

[30] I. Stojmenovic. Position based routing in ad hoc networks. IEEE Communications Magazine, 40(7):128-134, 2002. 